\title{
Average length of stay in Level III and IV Care of Accident and Emergency Department of Tertiary care Hospital
}

\author{
Reyaz A. Rangrez (Shah), M.D., ' Sheikh Mushtaq, M.B., ' Shafa Deva, M.D.,' Tanveer Rather, M.D., \\ S. Mufti, M.D., ${ }^{3}$ Abdul Majeed Ganai, M.D., ${ }^{3}$ Suzan Jalali, M.B. ${ }^{1}$ \\ Departments of ${ }^{1}$ Hospital Administration, ${ }^{2}$ Nuclear Medicine, and ${ }^{3}$ Community Medicine, Sher-i-Kashmir Institute of Medical Sciences \\ Srinagar
}

\section{A B S T R A C T}

BACKGROUND: The scope of Accident and Emergency (A\&E) Department is gradually broadening and in fact these are now becoming "mini hospitals within hospitals".

OBJECTIVE: To determine the average length of stay (LOS) in level III and level IV care and factors leading to prolonged length of stay. METHODS: A hospital based study with follow up of patients received in level III and level IV of A\&E Department and the patients were followed till transfer out to respective specialty wards, discharge or death.

RESULTS: Emergency beds occupied 9.3\% of the total hospital bed strength. Of the total emergency admissions studied, $71.1 \%$ comprised of neurosurgical admissions followed by CVTS (21.4\%), neurological (6.8\%) and other admissions (1.2\%). The average Length of Stay was greatest in CVTS followed by General Surgery i.e. 5.4 days and 4.6 days respectively. The time gap between investigations ordered and reports received was 1.04 days. $67 \%$ of the patients who attended A\&E Department were of rural background and out of it $54 \%$ have read up to high school.

CONCLUSIONS: Average Length of stay is 4.3 days which needs to be brought down to 24 hours as per international norms to provide equitable emergency care to wider population. Co-ordination between administration and clinicians is needed to expedite the problem. JMS $2011 ; 14(2): 61-62$

Key words: Length of stay, time gap, emergency care

Medical emergency is defined as a situation where the patient requires urgent and high quality medical care to prevent loss of life, limb or organ and initiate action for the restoration of normal healthy life. Accident is defined as an unexpected unplanned occurrence which may involve injury or an unprecedented event resulting in recognizable damage. ${ }^{1}$ The streamlined function of the Accident and Emergency Department goes a long way in projecting a good image of the hospital. "Accident and Emergency Department" name was recommended in 1961 by Platt Committee in UK. In India central council of health in 1963

Correspondence and reprint requests to:

Dr Reyaz A Rangrez (Shah)

Associate Professor

Department of Hospital Administration,

Sher-i-Kashmir Institute of Medical Sciences, Srinagar - 190011 urged all state Governments to set up Accident Emergency services. $^{2}$

Sher-i-Kashmir Institute of Medical Sciences (SKIMS) Srinagar is a 630 bedded tertiary care teaching Hospital and it has a well developed Accident and Emergency Department and separate super speciality departments. The Accident and Emergency Department has three levels of care; 1) Level I Filter Clinic, 2) Level II Emergency Care, and 3) Level III\&IV Emergency Recovery Wards. In addition 20 bedded Disaster ward is in close proximity to level III recovery ward. All the emergency patients are first received in Level I (Filter Clinic). Those requiring emergency admissions are admitted on a short stay admission protocol and observed at Level II for 6-24 hours before being discharged or transferred to respective super speciality care areas. Hospital besides receiving patients directly also receives patients referred from different hospitals across the valley and 
even patients outside valley are referred to this institute from time to time. This study was undertaken to determine the average length of stay at Level III \&IV of Accident and Emergency Department of the hospital and factors if any leading to prolonged Length of stay.

\section{Methods}

An observational, Prospective study was carried out for a period of six months from 1st January 2010 to 30th June 2010 at SKIMS. Though the main catchment area of the Hospital is Kashmir valley but it also receives patients across the state. The Hospital has a well developed Emergency care system. However, Orthopaedic, ENT and Eye Emergencies are referred to SKIMS associated Medical College and sometimes to Government Medical College associated Hospitals. All the patients admitted at level III and IV during the stated period were included in the study. The data was collected by scrutinizing the daily Census records, Inpatient case records and Electronic database of the Hospital. A predesigned questionnaire was set and observations were recorded in it on daily basis. Length of stay was defined as the total period from the date the patient was transferred to level III and IV to the transfer out/Discharge/Death from the level III and IV. The day of transfer out to progressive patient care area/Discharge was excluded. The data was statistically analyzed for the final outcome.

\section{Results}

The present study reveals that $9.3 \%$ of the total bed strength was occupied by Emergency admissions. $67 \%$ of the patients admitted in the Emergency were from rural background and 54 $\%$ of the patients admitted had read upto high school level. Neurosurgical and CVTS admissions accounted for majority of the patients $71.13 \%$ and $21.4 \%$ of the total surgical admissions respectively. This may be attributed to the fact that Neurosurgery and CVTS specialities are available at this Hospital only. On the medical side neurology and cardiology patients formed the main bulk who utilized accident and emergency services. 39\% of the total Emergency patients admitted in Accident and Emergency Department in surgical side overstayed beyond three days, where as in the medical side it was $31 \%$ of patients who overstayed beyond three days. The average length of stay was 4.3 days. It was highest in CVTS 5.4 days and lowest in Neurology 4.3 days. It was observed that more or less equal time gap existed between investigation ordered and specimen sent. The slight difference was because of type of investigation ordered. The time gap between specimen sent and investigation received was 1.4 days days. Bhadra \& Others in a study calculated average time gap of 3.33 days. ${ }^{3}$ Non emergency cases accounted for $1.04 \%$ of total admissions.

\section{Discussion}

From the above observations it could be concluded that average length of stay in level III and level IV of Accident and

\section{Table 1. Average length of stay in Surgical and Medical specialities}

\begin{tabular}{cc} 
Specialty & Average stay (days) \\
Surgical & $4.78 \pm 0.42^{*}$ \\
Neurosurgery & 4.5 \\
CVTS & 5.4 \\
Plastic Surgery & 4.6 \\
General Surgery & 4.6 \\
Medical & $4.73 \pm 0.31^{*}$ \\
General Medicine & 5.0 \\
Cardiology & 4.6 \\
Neurology & 4.3 \\
Medical Oncology & 4.5 \\
Clinical haematology & 5.0 \\
Nephrology & 5.0 \\
\hline
\end{tabular}

*(Mean \pm SD); $p$ (Surgical vs. Medical) $>0.8$

Emergency Department of SKIMS is 4.3 days which needs to be reduced to less than 24 hours so that emergency beds can be utilized for fresh patients attending the Accident Emergency Department. Close coordination, practice of evidence based medicine and discharge policy based on rational scientific and realistic approach could go a long way in solving this problem. Further research is needed to determine the factors leading to prolonged length of stay in progressive patient care areas. Sher-iKashmir Institute of Medical Sciences has excellent diagnostic investigation backup facilities and to decrease time gap between samples sent and reports received could be minimised by providing efficient logistic support. Huge rush of accident and emergency cases could be to some extent decreased by mobilizing equipment and human resource available in district level hospitals and by adopting a rational referral policy. A separate trauma care centre is the need of the hour.

\section{References}

1. Joshi SK. Quality Management in Hospitals. Jaypee Brothers Medical Publishers (P) Ltd. First edition: 2009. pp. 46-51.

2. Bhadra UK. An analysis of inpatient system of a state hospital in Calcutta; thesis submitted to AIIHPH Calcutta 1984 (unpublished).

3. IGNOU PGDHHMvol clinical services.

4. Sharma Y. Handbook of Hospital Administration 1st edition. Jammu: Durga Publications; 2005.

5. Thapa V, Saha JB, Ram R, Lahiri SK, Sarkar GN. A study of Some Facets Reflecting in-patient care of Naxalbari Rural Hospital on Indo-Nepal Border of West Bengal and the Quality of the Care as Perceived by the Patients. Indian Journal of Community Medicine 2001; 26(2):4-6. 